\title{
EVOLUTION OF A FERROFLUID FLOATING LAYER UNDER THE INFLUENCE OF AN INHOMOGENEOUS MAGNETIC FIELD
}

\author{
C.A. Bushueva, K.G. Kostarev, A.V. Lebedev \\ Institute of Continuous Media Mechanics \\ Perm, 1 Akad. Korolev Str., Russian Federation
}

\begin{abstract}
In this paper, we investigate the influence of a vertically aligned magnetic field of a circular current loop on a ferrofluid layer located on a liquid substrate. It has been found that an increase in field strength leads to a threshold formation of the layer rupture in the form of a regular circle. The critical value of the field strength depends linearly on the initial layer thickness. The rupture initiated by the magnetic field does not disappear after removal of the field provided that the thickness of the ferrofluid layer is lower than the critical value. In the case of deformation of rather thick layers the occurrence of rupture is accompanied by the formation of well-ordered drop structures.
\end{abstract}

Introduction. A stable rupture of the upper layer of a two-layer liquid system has been discovered quite recently [1]. A particular feature of such a system is that the layer rupture exists in the absence of any external force and has the shape of a perfect circle, inside which the fluid of the lower layer comes into contact with a gas. In [2], such a rupture occurred due to deformation of an initially continuous fluid layer caused by short-time application of some mechanical force or local injection of a microdose of a surfactant. It has been shown that the rupture is retained only in the case of a rather thin layer, whereas in thick layers the rupture closes as soon as the action of external factors ceases. In addition, the occurrence of a stable rupture can be expected when the upper fluid meets the following requirements: (a) it wets the walls of the cuvette, (b) it has a lower density $\rho$, and (c) it has a higher surface tension $\sigma$ compared to that of the lower fluid. With these restrictions we may create a two-layer system based on a ferrofluid and apply an inhomogeneous magnetic field to initiate a stable rupture.

1. Experimental technique. For the experiment we used a kerosene-based ferrofluid with dispersed magnetite particles stabilized by oleic acid. The main characteristics of the ferrofluid are: density $\rho_{1}=1.37 \mathrm{~g} / \mathrm{cm}^{3}$, surface tension $\sigma_{1}=$ $27 \mathrm{dyn} / \mathrm{cm}$, magnetic susceptibility $\chi=7.18$. For a liquid substrate, we chose perfluorooctane $\mathrm{C}_{8} \mathrm{~F}_{18}\left(\rho_{0}=1.76 \mathrm{~g} / \mathrm{cm}^{3}, \sigma_{0}=15.8 \mathrm{dyn} / \mathrm{cm}\right)$.

As a container for a two-layer system, we used a cuvette in the form of a short vertical cylinder made of borosilicate glass manufactured by JENAerGLAS. The thickness of the ferrofluid layer was defined as $h=m /\left(\rho_{2} S\right)$, where $m$ is the fluid mass, $S=D^{2} / 4$ is the area of the initially continuous layer, which is considered to be plane, $D$ is the inner diameter of the cuvette. The fluid mass and, accordingly, the layer thickness were determined by weighting a syringe with the ferrofluid on an electronic scales AnD EK 610i before and after its injection into the cuvette. In our experiments, the thickness of the ferrofluid layer varied from $1 \mathrm{~mm}$ to $5 \mathrm{~mm}$. The thickness of the liquid substrate was several times larger than the layer thickness (from 10 up to $20 \mathrm{~mm}$ ). 


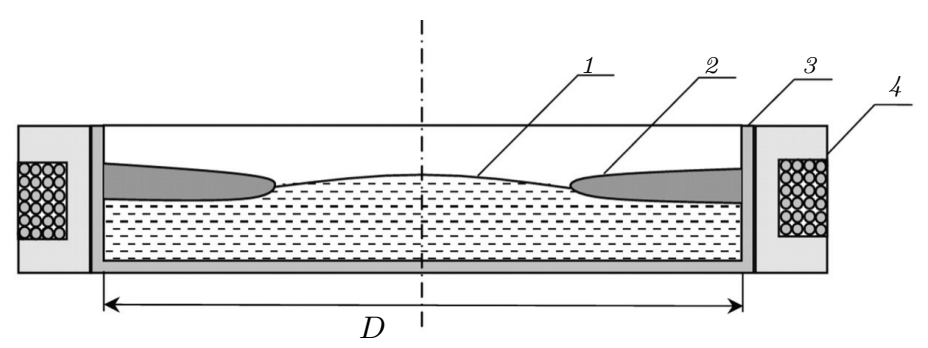

Fig. 1. Scheme of the experimental setup: 1 - perfluorooctane; 2 - ferrofluid; 3 cuvette; 4 - coil.

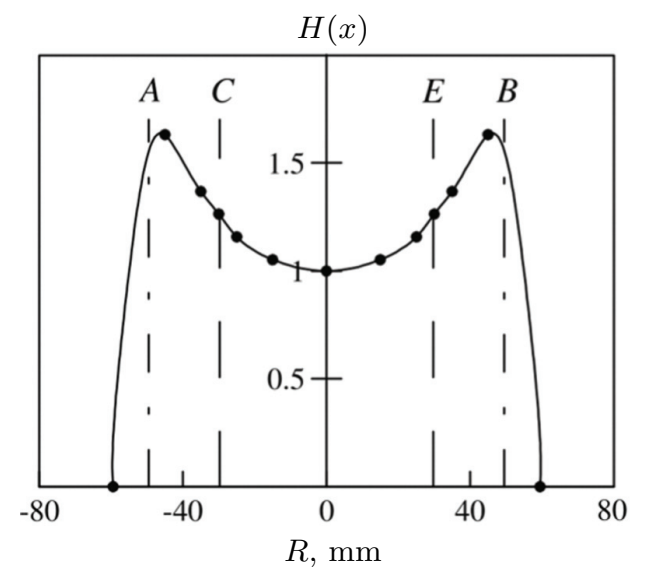

Fig. 2. Distribution of the relative vertical component of the field strength along the coil radius. Lines $A$ and $B, C$ and $E$ denote the boundaries of the cuvette with $D_{1}=92.6 \mathrm{~mm}$ and $D_{2}=59.4 \mathrm{~mm}$.

During the experiment, the cuvette with $D_{1}=92.6 \mathrm{~mm}$ or $D_{2}=59.4 \mathrm{~mm}$ is placed inside the horizontally located coil, whose inner diameter fits the outer diameter of the cuvette $D_{1}$ (Fig. 1).

The dimensions of the winding cross-sections were $8 \times 7 \mathrm{~mm}^{2}$. The winding was connected to a stabilized power supply source GPR $-7550 \mathrm{D}$. A relative distribution of the vertical component of the field strength along the coil radius located on the level of the free surface of the continuous non-deformed fluid layer is shown in Fig. 2. The field strength $H$ in the center of the coil will be used as its main characteristic.

The cuvette image was recorded by a video camera mounted above the coil. In addition, the frame field was provided with information about the strength of the electrical current passing through the coil. The experiment was carried out at ambient temperature $(24 \pm 1)^{\circ} \mathrm{C}$.

2. Results. Exposure of the ferrofluid to an inhomogeneous axisymmetric magnetic field of a circular current loop directed perpendicularly to the layer surface leads to redistribution of the ferrofluid along the cuvette radius. The layer thickness decreases at the center of the cuvette (in the region of the weakest field) and increases at the cuvette walls, where the field is stronger. At the critical value of the field strength $H^{*}$, the deformation penetrates through the whole layer and reaches the surface of the substrate. In this case, the layer rupture occurs in a threshold manner (shown in Fig. 3 by dashed vertical lines). 


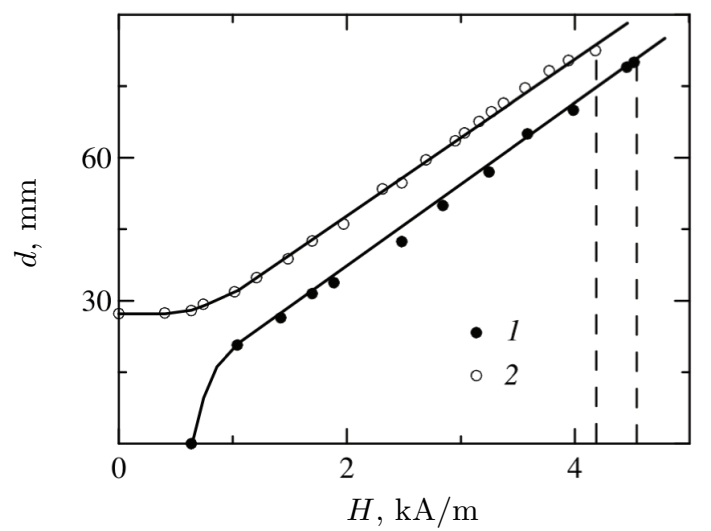

Fig. 3. Diameter of the initiated rupture of the ferrofluid layer $(D=92.6 \mathrm{~mm})$ versus the strength of the applied magnetic field. The thickness of the breaking layer $h: 2.7 \mathrm{~mm}$ (curve 1); $2.6 \mathrm{~mm}$ (curve 2).

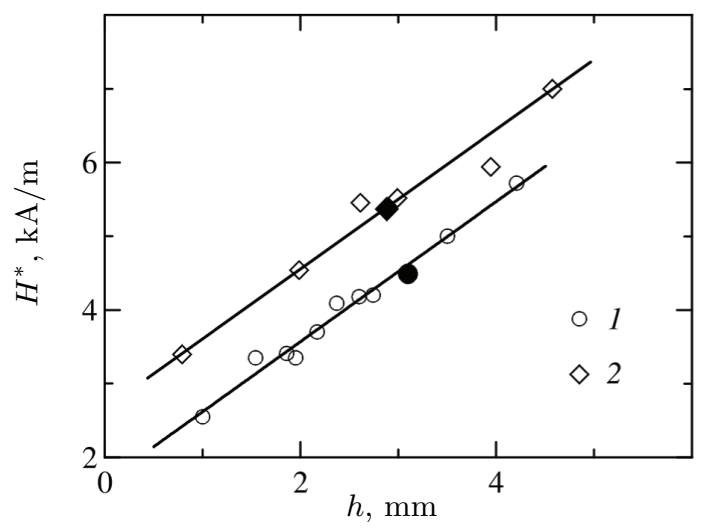

Fig. 4. Critical strength of the magnetic field versus the initial thickness of the breaking layer of the ferrofluid. The diameter of the cuvette $D: 92.6 \mathrm{~m}$ (curve 1), $59.4 \mathrm{~mm}$ (curve 2). Filled symbols on curves 1 and 2 correspond to the layers with $h=h_{\max }$.

In this figure one can see that for the layer of thickness $h=2.7 \mathrm{~mm}$ the rupture occurs at the field strength $H=4.5 \mathrm{kA} / \mathrm{m}$ and its diameter is approximately equals to $80 \mathrm{~mm}$. The growth of the field leads to an increase of the rupture diameter. If the field strength starts decreasing slowly, the rupture diameter gets smaller and smaller (Fig. 3, curve 1) until the rupture totally disappears at $H=0.7 \mathrm{kA} / \mathrm{m}$. It is worth saying that up to $H=1 \mathrm{kA} / \mathrm{m}$ the diameter of the rupture decreases almost linearly with the decreasing field strength, however, any further decrease of the field results in the abrupt collapse of the rupture.

Thus, there is a hysteresis in the process of rupture appearance in the increasing field and its closing in the decreasing field. This kind of behaviour can be explained both by a change of the surface topology after its rupture and by a change in the configuration of the magnetic field after the appearance of the layer rupture.

The layer rupture caused by the action of the magnetic field persists after the removal of the latter (Fig. 3, curve 2) provided that the initial thickness of the ferrofluid layer does not exceed $(85 \div 90) \%$ of $h_{\max }$, where $h_{\max }$ is the maximum thickness of the layer with a stable rupture of the layer surface [2]. 


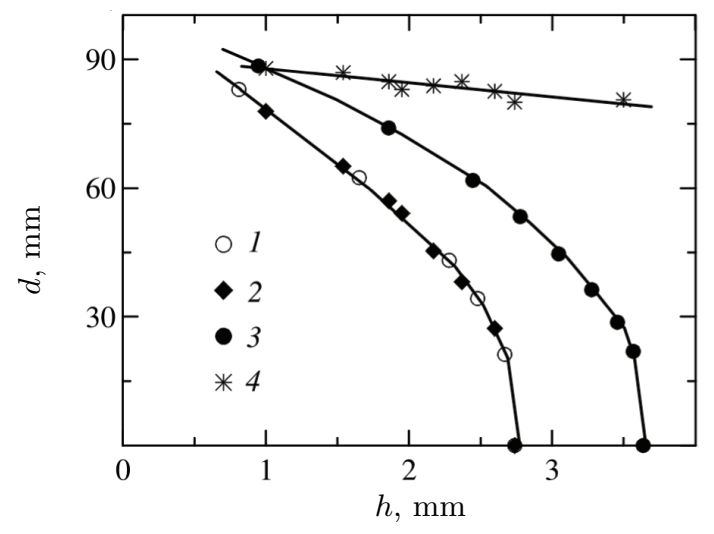

Fig. 5. Diameter of the rupture versus the layer thickness: in the absence of the field (curve 1), after the removal of the magnetic field (curve 2), in the field with $H=2.5 \mathrm{kA} / \mathrm{m}$ (curve 3), at the time of rupture formation during exposure to the magnetic field (curve 4). $D=92.6 \mathrm{~mm}$.

The critical field strength depends linearly on the thickness of the initial layer of the ferrofluid (Fig. 4). Filled symbols on curves 1 and 2 correspond to the layers with $h=h_{\max }$. Note that in a cuvette of a smaller diameter the rupture of layers of the same thickness occurs at a much higher field intensity. This can be explained by a decrease in inhomogeneity of the magnetic field along the radius of a smaller cuvette (the relative difference in field strength between the cuvette center and its periphery is no more than $30 \%$, whereas for a cuvette of a larger diameter this difference is $60 \%$, see Fig. 2).

Fig. 5 describes the dependence of the diameter of the stable rupture on the thickness of the initial ferrofluid layer for various cases of the presence/absence of a magnetic field. In particular, curves 1 and 4 in Fig. 5 describe the dependence $d(h)$ of the stable rupture in the absence of the magnetic field and after cessation of its action. Curve 1 was obtained for a case, when the layer of the ferrofluid was deformed by a narrow directional air jet until it broke, and curve 2 for a case, when the field strength was reduced to zero (see curve 2 in Fig. 3). The coincidence of these curves proves that the existence of stable rupture does not depend on the methods of its creation.

When the field strength does not reach the critical value, the vertical magnetic field becomes the factor, which facilitates the rupture of the ferrofluid layer. Thus, curve 3 in Fig. 5 illustrates the dependence of the diameter of the stable rupture produced by an air jet on the layer thickness at a fixed value of the field strength. A comparison between this curve and curve 1 suggests that the magnetic field can provoke rupture in a thicker layer.

Curve 4 in Fig. 5 describes the dependence $d(h)$ at the time of occurrence of the layer rupture under the action of the magnetic field. It is worth noting that a three-fold increase of the layer thickness causes only a $10 \%$ decrease in the rupture diameter. It has been found that the rupture of thicker layers occurs at an considerably stronger magnetic field (see Fig. 4). An increase in field intensity is naturally accompanied by a growth of the field gradient and, accordingly, by an increase of the magnetic ponderomotive forces attracting a larger volume of the ferrofluid. This is just the reason of why the rupture diameter depends weakly on the layer thickness. 

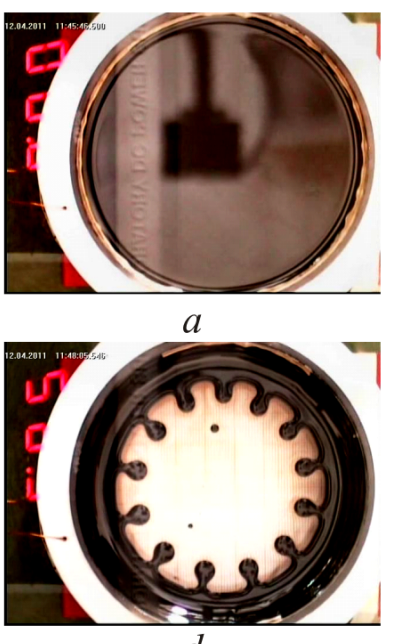

$d$

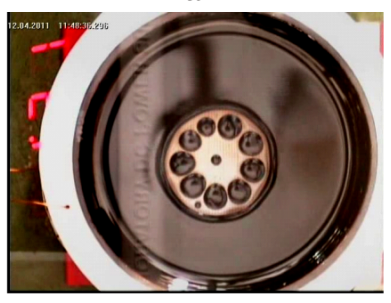

$h$
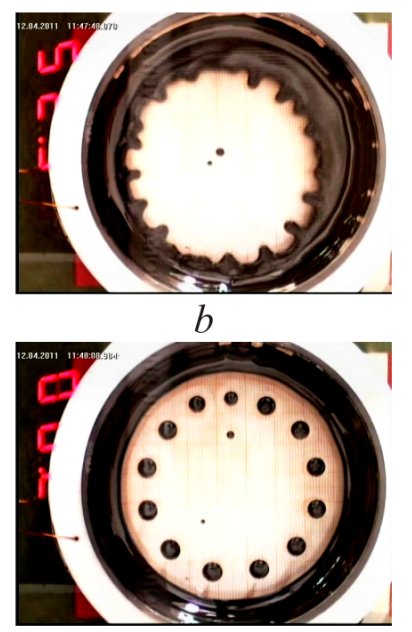

$e$

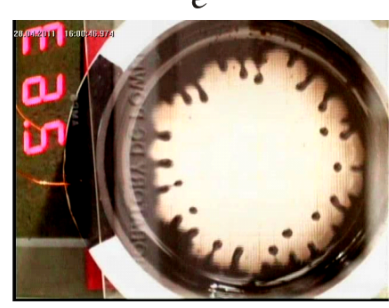

$i$
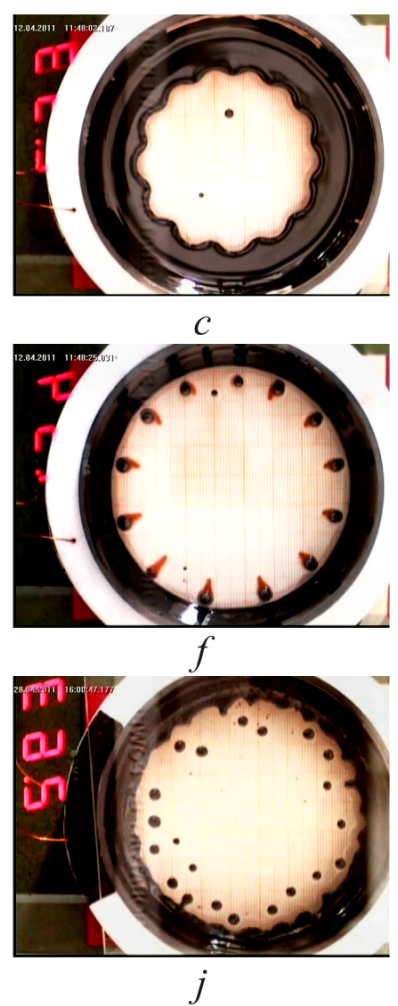

Fig. 6. Rupture of the ferrofluid layer of thickness $h=5.2 \mathrm{~mm}$ in the cuvette with $D=92.6 \mathrm{~mm}$. The field strength $H, \mathrm{kA} / \mathrm{m}$; the time elapsed since the beginning of the test $t$, s: $0,0(a) ; 5.3,118-132(b-c) ; 5.5,135(d) ; 6.4,138(e) ; 9.2,155(f) ; 4.0,166(h)$; $5.6,75-76(i-j)$. Closure of the layer at $3.5 \mathrm{kA} / \mathrm{m}$.

When the layer thickness exceeds $1.3 \times h_{\max }$ (reaching $4-5 \mathrm{~mm}$ ), the boundary of the initiated rupture becomes unstable. This instability may result from the inability of the magnetic field to hold the increasing volume of the ferrofluid within the near-wall region. The instability occurs at the stage of rupture formation, and the boundary of the rupture is perturbed periodically (Fig. 6b). Depending on the layer thickness, this disturbance may either decay, as it happens in the case of $h \sim 4.0 \div 4.3 \mathrm{~mm}$ in the cuvette with $D_{1}$, or evolve further (at $h \sim 4.5 \mathrm{~mm}$ ). In the latter case, the growth of the field intensity leads to an increase of the rupture diameter and to transformation of the boundary disturbance into a system of serrations (Fig. 6c,d). Then the serrations are detached in the form of drops, which form a well-ordered structure along the boundary of the rupture (Fig. 6e). With the growth of the field strength, the drops turn into cones, which are partially immersed in the liquid of the substrate and tend to migrate towards the wall meniscus (Fig. $6 f$ ). With the decreasing strength of the magnetic field, the area of the rupture reduces and the drops are pushed closer to each other (Fig. 6h). Then the rupture closes, still being under the action of the magnetic field of finite strength.

The number of drops arising at the rupture boundary depends on the thickness of the initial layer: at $h \sim 4.5 \mathrm{~mm}$ the number of drops varies from 5 to 9 , whereas at $h \sim 5.2 \mathrm{~mm}$ it increases to 13 . Moreover, the number of drops strongly depends on the field growth rate. In order to characterize the growth rate of the magnetic 


\section{C.A.Bushueva, K.G. Kostarev, A.V. Lebedev}

field, the viscous time of the system $\tau=h D / 4 \nu$, where $\nu$ is the kinematic viscosity of the ferrofluid, can be used. When the rupture formation in the layer of the same thickness occurs within a time period shorter than the viscous one $\tau$, the number of drops increases up to 20, but their size decreases (Fig. 6i,j).

The instability evolution in the cuvette with $D_{2}=59.4 \mathrm{~mm}$ follows a different scenario. Since this cuvette is exposed to a relatively homogeneous magnetic field, the occurrence of the rupture is accompanied by the formation of a drop structure like in the case of a vertically aligned magnetic field [3].

3. Conclusion. The experiments have shown that the exposure of the ferrofluid layer on a liquid substrate to a magnetic field of a circular current loop initiates its rupture. The rupture of the layer appears abruptly at a certain critical field strength. With the following decrease of the applied magnetic field, the rupture size exhibits a hysteresis, so that it decreases slowly with the field and might even remain finite when the field reaches zero. The value of the critical field strength responsible for the change in the topology of the layer is directly proportional to its thickness. The diameter of the initiated rupture depends weakly on the initial layer thickness and linearly on the field intensity.

Acknowledgements. The work was supported by the grant of the research program of the Ural Branch of RAS No. 09-T-1-1005 and the RFBR project No. 1002-96022.

\section{REFERENCES}

[1] Yu.Yu. Stollov. Oscillation of evaporating liquids and ispalator paradoxes. Phys. Usp., vol. 43 (2000), pp. 39-53 (in Russ.).

[2] Yu.K. Bratukhin, A.L. Zuev, K.G. Kostarev, A.V. Shmyrov. Stability of a steady-state discontinuity of a fluid layer on the surface of an immiscible fluid. Fluid Dynamics, vol. 44 (2009), no. 3, pp. 340-350.

[3] C.A. Bushueva, K.G. Kostarev. Dynamics of a ferrofluid layer with a stable rupture of the surface. Proc. the XXXVIII Summer School-Conference "Advanced Problems in Mechanics" (St. Petersburg, Russia, 2010), p. 98-104. 